ESAIM: M2AN 48 (2014) 583-602

DOI: $10.1051 / \mathrm{m} 2 \mathrm{an} / 2013105$
ESAIM: Mathematical Modelling and Numerical Analysis

www.esaim-m2an.org

\title{
SECOND-ORDER MUSCL SCHEMES BASED ON DUAL MESH GRADIENT RECONSTRUCTION (DMGR)
}

\author{
Christophe Berthon $^{1}$, Yves Coudière ${ }^{2,3}$ And Vivien Desveaux ${ }^{1}$
}

\begin{abstract}
We discuss new MUSCL reconstructions to approximate the solutions of hyperbolic systems of conservations laws on 2D unstructured meshes. To address such an issue, we write two MUSCL schemes on two overlapping meshes. A gradient reconstruction procedure is next defined by involving both approximations coming from each MUSCL scheme. This process increases the number of numerical unknowns, but it allows to reconstruct very accurate gradients. Moreover a particular attention is paid on the limitation procedure to enforce the required robustness property. Indeed, the invariant region is usually preserved at the expense of a more restrictive CFL condition. Here, we try to optimize this condition in order to reduce the computational cost.
\end{abstract}

Mathematics Subject Classification. 65M12, 35L65, 76M12.

Received July 5, 2012. Revised May 24, 2013.

Published online January 20, 2014.

\section{INTRODUCTION}

The present work is devoted to the numerical approximation of the weak solutions of nonlinear hyperbolic systems of conservation laws in two dimensions. The system under consideration reads

$$
\partial_{t} w+\partial_{x} f(w)+\partial_{y} g(w)=0, \quad t \geq 0, \quad(x, y) \in \mathbb{R}^{2},
$$

where $w: \mathbb{R}^{+} \times \mathbb{R}^{2} \rightarrow \Omega$ stands for the unknown state vector. Here, $\Omega \subset \mathbb{R}^{d}$ denotes the well-known set of admissible states and is assumed to be convex. Concerning the flux functions $f, g: \Omega \rightarrow \mathbb{R}^{d}$, as usual they are assumed to be smooth enough, at least Lipschitz-continuous.

In addition, we assume that the weak solutions satisfy the following entropy inequalities (see $[31,32])$

$$
\partial_{t} \eta(w)+\partial_{x} \mathcal{F}(w)+\partial_{y} \mathcal{G}(w) \leq 0
$$

where $w \mapsto \eta(w)$ is convex and $\mathcal{F}$ and $\mathcal{G}$ are the entropy flux functions and satisfy the following compatibility relations:

$$
\nabla f \nabla \eta=\nabla \mathcal{F}, \quad \nabla g \nabla \eta=\nabla \mathcal{G} .
$$

Keywords and phrases. Systems of conservation laws, MUSCL method, unstructured meshes, dual mesh, invariant region.

1 Laboratoire de Mathématiques Jean Leray, CNRS UMR 6629, Université de Nantes, 2 rue de la Houssinière, BP 92208,44322 Nantes Cedex 3, France. vivien.desveaux@univ-nantes.fr

2 Institut de Mathématiques de Bordeaux, CNRS UMR 5251, Université Bordeaux 1, 351 cours de la Libération, 33405 Talence Cedex, France.

3 INRIA Sud-Ouest, 351 cours de la Libération, 33405 Talence Cedex, France. 
During the few past decades, the derivation of numerical schemes to approximate the weak solutions of (1.1) was and remains a very active field and numerous methods are proposed in the literature. In the present paper, we focus on second-order finite volume schemes (see [23,33,43] and references therein) and we here discuss extensions of the well-known MUSCL scheme introduced by van Leer [44]. According to an a priori easy derivation of these approaches, the second-order MUSCL method turns out to be one of the most popular second-order finite volume procedure. Indeed, by considering a standard first-order finite volume scheme, van Leer [44] suggested to introduce a linear reconstruction of the approximated solution on each cell to evaluate the numerical flux function arguing these state reconstructions.

The gradient reconstructions are easily defined for $1 \mathrm{D}$ problems. We refer the reader to Leveque [33] (see also $[5,9,28,38])$ where several approaches are detailed. Of course, these 1D MUSCL reconstructions directly extend when 2D Cartesian meshes are involved. As soon as an unstructured mesh is considered, an important issue coming for the MUSCL scheme derivation turns out to be to correctly evaluate the prediction of the gradients. With triangles as control cells, several techniques of gradient reconstructions were introduced (see $[10-12,23,35,36])$ but do not easily extend to more general control cells like for cell-vertex schemes. One of the aim of the present work is to introduce a gradient reconstruction technique free from the definition of the control cells. To address such an issue, we suggest a suitable extension of the Discrete Duality Finite Volume (DDFV) methods $[2,21,25,26]$ to consider nonlinear hyperbolic systems.

In fact, the linear reconstruction procedure turns out to be a crucial point since spurious oscillations may occur. To avoid such numerical nuisances, a limitation procedure must be considered. A wide literature is devoted to the derivation of relevant limitations. For a non-exhaustive state of the art, the reader is referred for instance to the following works: $[3,5,7-11,13,18,19,22,27,29,34,37,45]$. In $[3,19,45]$, the main objective is the derivation of genuinely 2D limiters to get relevant reconstructions on unstructured meshes. Such an approach may enforce too much numerical viscosity in the regions where the solutions are smooth. Authors propose some additional limiter to artificially reduce the numerical viscosity (see $[29,37]$ ). To enforce some stability properties, articles are devoted to modify usual limiters to satisfy maximum principle statements (for instance $[4,27,34]$ ). In these works, the limiter is based on one gradient reconstruction over each cell. Another strategy consists in introducing one scalar slope per edge within each cell (see [10,13]).

The limitation is a key-point to ensure some robustness properties. Indeed, the MUSCL extension is not able to restore the invariance of $\Omega$ and a specific attention must be paid on the limitation derivation to ensure this essential property. Several strategies were introduced during the last decade. For instance, in $[7,18,38-40]$ the authors introduced a suitable gradient limitation to enforce the required robustness under a restrictive CFL condition. More recently, to avoid the CFL restriction, in [14,20], it is suggested an a posteriori limitation by imposing a vanishing gradient reconstruction as soon as the robustness is lost. Such a technique makes the scheme $\Omega$-preserving but at the cost of a locally lost of accuracy. As a consequence, it appears that enforcing robustness of second-order MUSCL schemes needs, in general, more restrictive CFL and therefore induces larger computational cost. Currently, it seems impossible to avoid such additional CFL restriction coming from the MUSCL procedure and we have to deal with it. Moreover, one of the goal of the present paper is to optimize this restriction. From now on, let us mention that the CFL condition is issued from both CFL number of the associated first-order scheme and the MUSCL robustness. Considering 2D unstructured meshes, we will establish that the usual first-order CFL condition is not optimal at all (for instance see [40]), introducing a non-relevant CFL multiplying factor which is sometime large. As a consequence, involving a rigorous first-order CFL condition may produce an excessive computation cost. Here, we will exhibit an optimal first-order CFL condition.

The paper is organized as follows. In the next Section we introduce the main notations and we briefly recall the derivation of second-order MUSCL schemes for $2 \mathrm{D}$ unstructured meshes. A particular attention is paid to the $\Omega$-preserving property which must be satisfied by the scheme and we recall the CFL restriction to ensure the required robustness. We conclude this section by exhibiting an optimal CFL condition coming for the announced first-order scheme. Section 3 deals with the derivation of the Dual Mesh Gradient Reconstruction. This approach is supplemented by a limitation procedure in order to enforce the $\Omega$-invariant preserving property. 


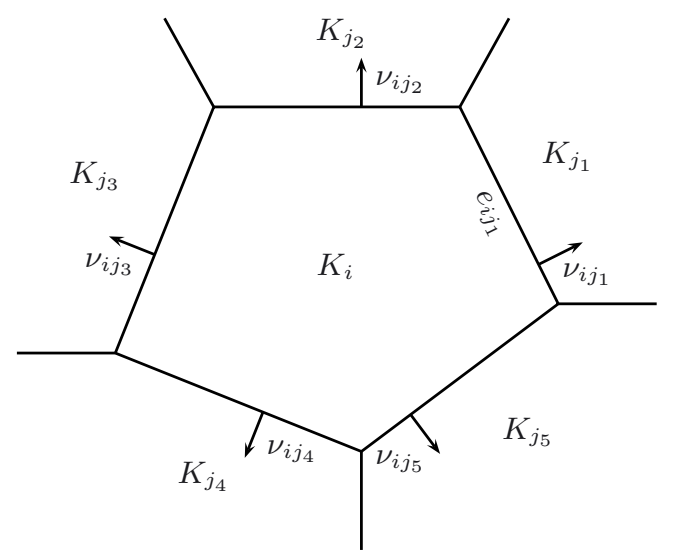

FiguRE 1. Geometry of the cell $K_{i}$.

Next, Section 4 is devoted to the numerical experiments. Finally, the paper is concluded in Section 5 with some comments and perspectives.

\section{MAin NOTATIONS AND MUSCL SCHEME}

For the sake of completeness, we recall the description of the MUSCL scheme for a 2D unstructured mesh, and we follow the usual approach (for instance see [23,39]). Next we focus on robustness of the method and we present new CFL conditions which are less restrictive than usual.

\subsection{MUSCL schemes}

First, we introduce the main notations to describe the considered unstructured mesh. Let us assume a mesh made of polygonal control cells $\left(K_{i}\right)_{i \in \mathbb{Z}}$. We denote by $\gamma(i)$ the index set of the cells neighbouring $K_{i}$. For all $j \in \gamma(i)$, we set $e_{i j}$ the common edge which separates $K_{i}$ and $K_{j}$, and $\nu_{i j}$ the unit outward normal to $e_{i j}$ (see Fig. 1). To simplify the notations, we introduce $\left|K_{i}\right|$ the area of $K_{i}$ and $\mathcal{P}_{i}=\sum_{j \in \gamma(i)}\left|e_{i j}\right|$ its perimeter, where $\left|e_{i j}\right|$ denotes the length of $e_{i j}$.

Now, on each cell $K_{i}$ at time $t^{n}, w_{i}^{n}$ stands for an approximation of the exact solution of the system (1.1). To obtain an approximation at time $t^{n+1}=t^{n}+\Delta t$, the sequence $\left(w_{i}^{n}\right)_{i \in \mathbb{Z}}$ evolves in time as follows:

$$
w_{i}^{n+1}=w_{i}^{n}-\frac{\Delta t}{\left|K_{i}\right|} \sum_{j \in \gamma(i)}\left|e_{i j}\right| \varphi\left(w_{i j}, w_{j i}, \nu_{i j}\right),
$$

where $w_{i j}$ and $w_{j i}$, assumed to be in $\Omega$, are second-order approximations of the solution at the middle of the edge $e_{i j}$ on each side (see Fig. 2). The reconstruction procedure to obtain both $w_{i j}$ and $w_{j i}$ is the purpose of the next section. Concerning the numerical flux function, as usual we assume $\varphi$ to be Lipschitz-continuous, consistent and conservative (see $[23,33,43]$ and references therein).

In the present work, in order to establish an optimal CFL condition, we restrict our analysis to Godunovtype numerical flux functions according to the work by Harten, Lax and van Leer [24]. Hence we introduce a one dimensional approximate Riemann solver $\widetilde{w}_{\nu}\left(\frac{x}{t} ; w_{L}, w_{R}\right)$ assumed to belong to $\Omega$ for $t>0$ and $x \in \mathbb{R}$, defined by

$$
\frac{1}{\delta} \int_{-\frac{\delta}{2}}^{\frac{\delta}{2}} \widetilde{w}_{\nu}\left(\frac{x}{t} ; w_{L}, w_{R}\right) \mathrm{d} x=\frac{1}{2}\left(w_{L}+w_{R}\right)-\frac{\Delta t}{\delta}\left(h_{\nu}\left(w_{R}\right)-h_{\nu}\left(w_{L}\right)\right),
$$




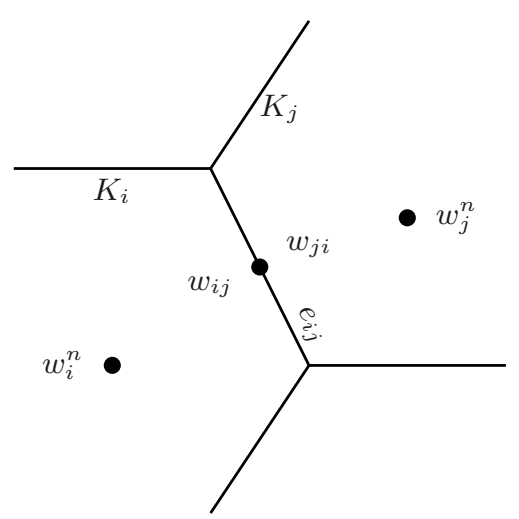

Figure 2. First-order states $w_{i}^{n}$ and $w_{j}^{n}$ and second-order reconstructed states $w_{i j}$ and $w_{j i}$.

where $h_{\nu}: \Omega \rightarrow \mathbb{R}^{d}$ defines the flux function in the $\nu$-direction as follows:

$$
h_{\nu}(w)=\nu_{x} f(w)+\nu_{y} g(w), \text { with } \nu=\left(\nu_{x}, \nu_{y}\right)^{\mathrm{T}} .
$$

From [24], the relation (2.2) is relevant as long as the time step $\Delta t$ is restricted according to the following CFL condition:

$$
\frac{\Delta t}{\delta} \max \left|\lambda^{ \pm}\left(w_{L}, w_{R}, \nu\right)\right| \leq \frac{1}{2}
$$

where $\lambda^{ \pm}\left(w_{L}, w_{R}, \nu\right)$ stand for both maximum and minimum characteristic speeds of the approximate Riemann solver $\widetilde{w}_{\nu}\left(\frac{x}{t} ; w_{L}, w_{R}\right)$. As mentioned in [24], let us note that we naturally impose the fan involved by the exact Riemann solution of the following 1D hyperbolic system of conservation laws:

$$
\left\{\begin{array}{l}
\partial_{t} w+\partial_{x} h_{\nu}(w)=0 \\
w(x, 0)=\left\{\begin{array}{l}
w_{L} \text { if } x<0 \\
w_{R} \text { if } x>0
\end{array}\right.
\end{array}\right.
$$

to be included into the fan coming from $\widetilde{w}_{\nu}\left(\frac{x}{t} ; w_{L}, w_{R}\right)$.

We are now able to give the 2D numerical flux function:

$$
\varphi\left(w_{L}, w_{R}, \nu\right)=h_{\nu}\left(w_{L}\right)+\frac{\delta}{2 \Delta t} w_{L}-\frac{1}{\Delta t} \int_{-\frac{\delta}{2}}^{0} \widetilde{w}_{\nu}\left(\frac{x}{\Delta t}, w_{L}, w_{R}\right) \mathrm{d} x .
$$

Arguing such a definition, we easily have the following statement:

Lemma 2.1. Assume $\widetilde{w}_{\nu}\left(\frac{x}{t} ; w, w\right)=w$ for all $w \in \Omega$. Then the numerical flux function $(2.4)$ is consistent:

$$
\varphi(w, w, \nu)=h_{\nu}(w), \text { for all } w \in \Omega .
$$

In addition, let us impose that the following relation holds:

$$
\int_{-\frac{\delta}{2}}^{0} \widetilde{w}_{\nu}\left(\frac{x}{t} ; w_{L}, w_{R}\right) \mathrm{d} x=\int_{0}^{\frac{\delta}{2}} \widetilde{w}_{-\nu}\left(\frac{x}{t} ; w_{R}, w_{L}\right) \mathrm{d} x,
$$

then the numerical flux function is conservative in the following sense:

$$
\varphi\left(w_{L}, w_{R}, \nu\right)=-\varphi\left(w_{R}, w_{L},-\nu\right) .
$$

We skip the proof which is obvious. 
Remark 2.2. From now on, let us emphasize that the numerical flux function $\varphi$, given by (2.4), is defined independently from the parameter $\delta$. Indeed, since we have

$$
\int_{-\frac{\delta}{2}}^{0} \widetilde{w}_{\nu}\left(\frac{x}{\Delta t}, w_{L}, w_{R}\right) \mathrm{d} x=\left(\frac{\delta}{2}+\lambda^{-}\left(w_{L}, w_{R}, \nu\right)_{-} \Delta t\right) w_{L}+\int_{\lambda^{-}\left(w_{L}, w_{R}, \nu\right)_{-} \Delta t}^{0} \widetilde{w}_{\nu}\left(\frac{x}{\Delta t}, w_{L}, w_{R}\right) \mathrm{d} x,
$$

where $x_{-}=\min (0, x)$, the numerical flux function rewrites

$$
\varphi\left(w_{L}, w_{R}, \nu\right)=h_{\nu}\left(w_{L}\right)+\lambda^{-}\left(w_{L}, w_{R}, \nu\right)_{-} w_{L}-\frac{1}{\Delta t} \int_{\lambda^{-}\left(w_{L}, w_{R}, \nu\right)_{-} \Delta t}^{0} \widetilde{w}_{\nu}\left(\frac{x}{\Delta t}, w_{L}, w_{R}\right) \mathrm{d} x .
$$

As expected, $\delta$ will characterize a mesh length definition. A precise definition of $\delta$ will be given later on to get an optimal CFL condition.

Moreover, once again following [24], we impose the approximate Riemann solver $\widetilde{w}_{\nu}$ to be consistent with the entropy inequalities (1.2) as follows:

$$
\frac{1}{\delta} \int_{-\frac{\delta}{2}}^{\frac{\delta}{2}} \eta\left(\widetilde{w}_{\nu}\left(\frac{x}{t} ; w_{L}, w_{R}\right)\right) \mathrm{d} x \leq \frac{1}{2}\left(\eta\left(w_{L}\right)+\eta\left(w_{R}\right)\right)-\frac{\Delta t}{\delta}\left(\mathcal{H}_{\nu}\left(w_{R}\right)-\mathcal{H}_{\nu}\left(w_{L}\right)\right),
$$

where $\mathcal{H}_{\nu}: \Omega \rightarrow \mathbb{R}^{d}$ defines the entropy flux function in the $\nu$-direction given by:

$$
\mathcal{H}_{\nu}(w)=\nu_{x} \mathcal{F}(w)+\nu_{y} \mathcal{G}(w) \text {, with } \nu=\left(\nu_{x}, \nu_{y}\right)^{\mathrm{T}} .
$$

This leads to a natural definition of the entropy numerical flux function

$$
\Phi\left(w_{L}, w_{R}, \nu\right)=\mathcal{H}_{\nu}\left(w_{L}\right)+\frac{\delta}{2 \Delta t} \eta\left(w_{L}\right)-\frac{1}{\Delta t} \int_{-\frac{\delta}{2}}^{0} \eta\left(\widetilde{w}_{\nu}\left(\frac{x}{\Delta t}, w_{L}, w_{R}\right)\right) \mathrm{d} x
$$

We can now prove an extension of Lemma 2.1 concerning the entropy:

Lemma 2.3. Assume $\widetilde{w}_{\nu}\left(\frac{x}{t} ; w, w\right)=w$ for all $w \in \Omega$. Then the entropy numerical flux function (2.8) is consistent:

$$
\Phi(w, w, \nu)=\mathcal{H}_{\nu}(w), \text { for all } w \in \Omega .
$$

In addition, let us impose that the following relation holds:

$$
\int_{-\frac{\delta}{2}}^{0} \eta\left(\widetilde{w}_{\nu}\left(\frac{x}{t} ; w_{L}, w_{R}\right)\right) \mathrm{d} x=\int_{0}^{\frac{\delta}{2}} \eta\left(\widetilde{w}_{-\nu}\left(\frac{x}{t} ; w_{R}, w_{L}\right)\right) \mathrm{d} x
$$

then the entropy numerical flux function satisfies the following property

$$
\Phi\left(w_{L}, w_{R}, \nu\right)+\Phi\left(w_{R}, w_{L},-\nu\right) \geq 0 .
$$

Proof. The consistency is easily proven thanks to (2.8). To establish the property (2.10), relations (2.8) and (2.9) give

which implies

$$
\Phi\left(w_{L}, w_{R}, \nu\right)=\mathcal{H}_{\nu}\left(w_{L}\right)+\frac{\delta}{2 \Delta t} \eta\left(w_{L}\right)-\frac{1}{\Delta t} \int_{0}^{\frac{\delta}{2}} \eta\left(\widetilde{w}_{-\nu}\left(\frac{x}{\Delta t}, w_{R}, w_{L}\right)\right) \mathrm{d} x,
$$

$$
\begin{array}{r}
\Phi\left(w_{L}, w_{R}, \nu\right)+\Phi\left(w_{R}, w_{L},-\nu\right)=\mathcal{H}_{\nu}\left(w_{L}\right)+\mathcal{H}_{-\nu}\left(w_{R}\right)+\frac{\delta}{2 \Delta t} \eta\left(w_{L}\right)+\frac{\delta}{2 \Delta t} \eta\left(w_{R}\right) \\
-\frac{1}{\Delta t} \int_{-\frac{\delta}{2}}^{\frac{\delta}{2}} \eta\left(\widetilde{w}_{-\nu}\left(\frac{x}{\Delta t}, w_{R}, w_{L}\right)\right) \mathrm{d} x .
\end{array}
$$

Using the inequality (2.7) and the exact flux property $\mathcal{H}_{-\nu}=-\mathcal{H}_{\nu}$, we obtain (2.10). 
Remark 2.4. Let us notice that in Lemma 2.3, (2.9) has to be satisfied for all entropy $\eta$, which is a priori a strong condition. However the pointwise conservation property

$$
\widetilde{w}_{\nu}\left(\frac{x}{t}, w_{L}, w_{R}\right)=\widetilde{w}_{-\nu}\left(-\frac{x}{t}, w_{R}, w_{L}\right) \text {, for all } x \in \mathbb{R} \text { and } t>0,
$$

implies both relations (2.5) and (2.9), and it is satisfied by the classical Riemann solvers (Godunov, HLL, Suliciu relaxation for Euler equations for instance, see [8,15, 23, 24]).

\subsection{A positive preserving first-order scheme}

Before we study the robustness of the scheme (2.1), we propose to analyse the CFL condition, called first-order CFL condition, to be satisfied by the associated first-order scheme:

$$
w_{i}^{n+1}=w_{i}^{n}-\frac{\Delta t}{\left|K_{i}\right|} \sum_{j \in \gamma(i)}\left|e_{i j}\right| \varphi\left(w_{i}^{n}, w_{j}^{n}, \nu_{i j}\right),
$$

which is obtained by enforcing $w_{i j}=w_{i}^{n}$ in $(2.1)$.

By considering the numerical flux given by (2.4) in the following statement, we propose to exhibit an optimal first-order CFL condition.

Theorem 2.5. For all $w_{L}$ and $w_{R}$ in $\Omega$, assume that $\widetilde{w}_{\nu}\left(\frac{x}{t} ; w_{L}, w_{R}\right)$ stays in $\Omega$. Let $w_{i}^{n}$ belongs to $\Omega$ for all $i \in \mathbb{Z}$. Assume the following CFL restriction:

$$
\Delta t \frac{\mathcal{P}_{i}}{\left|K_{i}\right|} \max _{j \in \gamma(i)}\left|\lambda^{ \pm}\left(w_{i}^{n}, w_{j}^{n}, \nu_{i j}\right)\right| \leq 1, \quad \forall i \in \mathbb{Z} .
$$

Then the updated states $w_{i}^{n+1}$, given by $(2.12)$ remain in $\Omega$.

In addition, the scheme (2.12) satisfies the following entropy inequalities:

$$
\eta\left(w_{i}^{n+1}\right) \leq \eta\left(w_{i}^{n}\right)-\frac{\Delta t}{\left|K_{i}\right|} \sum_{j \in \gamma(i)}\left|e_{i j}\right| \Phi\left(w_{i}^{n}, w_{j}^{n}, \nu_{i j}\right)
$$

Proof. First, let us note that both CFL conditions (2.3) and (2.13) coincide as soon as $w_{L}=w_{i}^{n}, w_{R}=w_{j}^{n}$ and $\delta=\frac{2\left|K_{i}\right|}{\mathcal{P}_{i}}$. As a consequence, the integral formulation of the numerical flux (2.4) can be adopted:

$$
\varphi\left(w_{i}^{n}, w_{j}^{n}, \nu_{i j}\right)=h_{\nu_{i j}}\left(w_{i}^{n}\right)+\frac{\left|K_{i}\right|}{\mathcal{P}_{i} \Delta t} w_{i}^{n}-\frac{1}{\Delta t} \int_{-\frac{\left|K_{i}\right|}{\mathcal{P}_{i}}}^{0} \widetilde{w}_{\nu_{i j}}\left(\frac{x}{\Delta t}, w_{i}^{n}, w_{j}^{n}\right) \mathrm{d} x .
$$

Now, by involving the Green formula, let us recall that $\sum_{j \in \gamma(i)}\left|e_{i j}\right| h_{\nu_{i j}}\left(w_{i}^{n}\right)=0$. Then, by plugging (2.15) into (2.12), we obtain

$$
w_{i}^{n+1}=\frac{1}{\left|K_{i}\right|} \sum_{j \in \gamma(i)}\left|e_{i j}\right| \int_{-\frac{\left|K_{i}\right|}{\mathcal{P}_{i}}}^{0} \widetilde{w}_{\nu_{i j}}\left(\frac{x}{\Delta t}, w_{i}^{n}, w_{j}^{n}\right) \mathrm{d} x .
$$

Since $\widetilde{w}_{\nu_{i j}}\left(\frac{x}{\Delta t}, w_{i}^{n}, w_{j}^{n}\right) \in \Omega$ and $\Omega$ is a convex set, we immediately deduce that

$$
\widehat{w}_{i j}:=\frac{\mathcal{P}_{i}}{\left|K_{i}\right|} \int_{-\frac{\left|K_{i}\right|}{\mathcal{P}_{i}}}^{0} \widetilde{w}_{\nu_{i j}}\left(\frac{x}{\Delta t}, w_{i}^{n}, w_{j}^{n}\right) \mathrm{d} x \in \Omega .
$$

Finally, the state $w_{i}^{n+1}$ stands for a convex combination of states in $\Omega$ and thus the $\Omega$-preserving property is established. 
To prove the entropy inequalities, we use the convexity of $\eta$ and the Jensen inequality in equation (2.16) to get

$$
\eta\left(w_{i}^{n+1}\right) \leq \frac{1}{\left|K_{i}\right|} \sum_{j \in \gamma(i)}\left|e_{i j}\right| \int_{-\frac{\left|K_{i}\right|}{\mathcal{P}_{i}}}^{0} \eta\left(\widetilde{w}_{\nu_{i j}}\left(\frac{x}{\Delta t}, w_{i}^{n}, w_{j}^{n}\right)\right) \mathrm{d} x .
$$

Involving the Green formula, we have

$$
\eta\left(w_{i}^{n}\right)-\frac{\Delta t}{\left|K_{i}\right|} \sum_{j \in \gamma(i)}\left|e_{i j}\right|\left(\mathcal{H}_{\nu_{i j}}\left(w_{i}^{n}\right)+\frac{\left|K_{i}\right|}{\Delta t \mathcal{P}_{i}} \eta\left(w_{i}^{n}\right)\right)=0 .
$$

Summing equations (2.17) and (2.18), and using the definition (2.8), we get exactly the expected entropy inequalities (2.14).

To conclude this section devoted to the presentation of the adopted first-order scheme, let us emphasize that similar 2D techniques can be found in the literature (for instance see [40]). In general, a specific attention is paid to the CFL condition and several works $[39,40]$ involve a half-CFL number. However, it is important to note that this too restrictive time step easily increases to get the CFL condition (2.13) as soon as the numerical flux function comes from an approximate Riemann solver. Moreover, let us notice that the CFL condition (2.13) coincides with the one proposed in [7] as soon as the cells are nothing but regular polygons. An easy calculation shows that the derived CFL condition (2.13) is less restrictive than the one given in [7] when the cells turn out to be non-regular polygons.

\subsection{Robustness of the MUSCL scheme}

The objective is now to extend Theorem 2.5 to second-order MUSCL scheme. To obtain such a result, we adopt classical decomposition of the MUSCL scheme as a convex combination of first-order schemes (for instance see $[7,39])$. As a consequence, the CFL restriction of the MUSCL scheme is strongly related to the first-order one (2.13).

We first need to introduce further notations. Let $G_{i}$ be the mass center of the cell $K_{i}$ and for all $j \in \gamma(i)$, we denote by $T_{i j}$ the triangle formed by the point $G_{i}$ and the edge $e_{i j}$. These triangles are essential since these are the subcells on which we will apply Theorem 2.5. Let $\gamma(i, j)$ be the index set of the two subcells neighbouring $T_{i j}$ in the cell $K_{i}$. For all $k \in \gamma(i, j)$, we set $e_{j k}^{i}$ the common edge which separate $T_{i j}$ and $T_{i k}$ and $\nu_{j k}^{i}$ the unit outward normal to $e_{j k}^{i}$ (see Fig. 3). Finally, we denote respectively by $\left|T_{i j}\right|, \mathcal{P}_{i j}$ and $\left|e_{i j}\right|$, the area of the subcell $T_{i j}$, its perimeter and the length of the edge $e_{i j}$.

Arguing these notations, we are able to establish the robustness of the second-order MUSCL scheme.

Theorem 2.6. For all $w_{L}$ and $w_{R}$ in $\Omega$, assume that $\widetilde{w}_{\nu}\left(\frac{x}{t} ; w_{L}, w_{R}\right)$ stays in $\Omega$. Let the average states $w_{i}^{n}$ and the reconstructed states $w_{i j}$ belong to $\Omega$ for all $i \in \mathbb{Z}$ and for all $j \in \gamma(i)$. Assume the reconstructed states satisfy the following conservation property:

$$
\sum_{j \in \gamma(i)} \frac{\left|T_{i j}\right|}{\left|K_{i}\right|} w_{i j}=w_{i}^{n}
$$

If the CFL restriction

$$
\Delta t \max _{j \in \gamma(i)} \frac{\mathcal{P}_{i j}}{\left|T_{i j}\right|} \max _{k \in \gamma(i, j)}\left\{\lambda^{ \pm}\left(w_{i j}, w_{j i}, \nu_{i j}\right), \lambda^{ \pm}\left(w_{i j}, w_{i k}, \nu_{j k}^{i}\right)\right\} \leq 1, \quad \forall i \in \mathbb{Z},
$$

is satisfied, then the updated states $w_{i}^{n+1}$, given by (2.1), remain in $\Omega$.

In addition, the scheme (2.1) satisfies the following entropy inequalities:

$$
\eta\left(w_{i}^{n+1}\right) \leq \bar{\eta}_{i}^{n}-\frac{\Delta t}{\left|K_{i}\right|} \sum_{j \in \gamma(i)}\left|e_{i j}\right| \Phi\left(w_{i j}, w_{j i}, \nu_{i j}\right),
$$




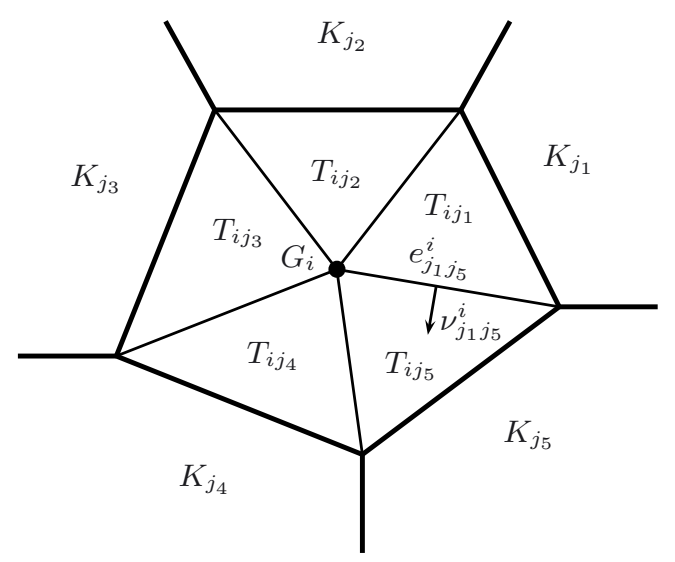

FIGURE 3. Subcells decomposition of the cell $K_{i}$.

where $\bar{\eta}_{i}^{n}$ is defined as follows:

$$
\bar{\eta}_{i}^{n}=\sum_{j \in \gamma(i)} \frac{\left|T_{i j}\right|}{\left|K_{i}\right|} \eta\left(w_{i j}\right) .
$$

Proof. At time $t^{n}$, let us assume that we know a piecewise constant approximate solution whose value on the subcell $T_{i j}$ is $w_{i j}$. We evolve this solution by the first-order scheme (2.12) until time $t^{n+1}$ and we get a state $w_{i j}^{n+1}$ on the subcell $T_{i j}$, given by

$$
w_{i j}^{n+1}=w_{i j}-\frac{\Delta t}{\left|T_{i j}\right|}\left(\left|e_{i j}\right| \phi\left(w_{i j}, w_{j i}, \nu_{i j}\right)+\sum_{k \in \gamma(i, j)}\left|e_{j k}^{i}\right| \phi\left(w_{i j}, w_{i k}, \nu_{j k}^{i}\right)\right) .
$$

Since the CFL condition (2.20) implies (2.13), Theorem 2.5 can be applied to ensure that the state $w_{i j}^{n+1}$ is in $\Omega$. Next, arguing the flux conservation property (2.6), we easily establish the following relation satisfied by the intermediate states $w_{i j}^{n+1}$ :

$$
\frac{1}{\left|K_{i}\right|} \sum_{j \in \gamma(i)}\left|T_{i j}\right| w_{i j}^{n+1}=\sum_{j \in \gamma(i)} \frac{\left|T_{i j}\right|}{\left|K_{i}\right|} w_{i j}-\frac{\Delta t}{\left|K_{i}\right|} \sum_{j \in \gamma(i)}\left|e_{i j}\right| \phi\left(w_{i j}, w_{j i}, \nu_{i j}\right) .
$$

Next, involving the conservation assumption (2.19) and the definition of second-order scheme (2.1), we immediately obtain

$$
w_{i}^{n+1}=\frac{1}{\left|K_{i}\right|} \sum_{j \in \gamma(i)}\left|T_{i j}\right| w_{i j}^{n+1}
$$

Since $w_{i}^{n+1}$ is nothing but a convex combination of states in $\Omega$, we deduce that $w_{i}^{n+1}$ belongs to $\Omega$.

In order to establish the entropy inequalities (2.21)-(2.22), the second part of Theorem 2.5 ensures that we have

$$
\eta\left(w_{i j}^{n+1}\right) \leq \eta\left(w_{i j}\right)-\frac{\Delta t}{\left|T_{i j}\right|}\left(\left|e_{i j}\right| \Phi\left(w_{i j}, w_{j i}, \nu_{i j}\right)+\sum_{k \in \gamma(i, j)}\left|e_{j k}^{i}\right| \Phi\left(w_{i j}, w_{i k}, \nu_{j k}^{i}\right)\right) .
$$

The discrete Jensen inequality applied to equation (2.23) immediately gives

$$
\eta\left(w_{i}^{n+1}\right) \leq \frac{1}{\left|K_{i}\right|} \sum_{j \in \gamma(i)}\left|T_{i j}\right| \eta\left(w_{i j}^{n+1}\right),
$$


to deduce

$$
\eta\left(w_{i}^{n+1}\right) \leq \eta_{i}^{n}-\frac{\Delta t}{\left|K_{i}\right|} \sum_{j \in \gamma(i)}\left|e_{i j}\right| \Phi\left(w_{i j}, w_{j i}, \nu_{i j}\right)-\frac{\Delta t}{\left|K_{i}\right|} \sum_{j \in \gamma(i)} \sum_{k \in \gamma(i, j)}\left|e_{j k}^{i}\right| \Phi\left(w_{i j}, w_{i k}, \nu_{j k}^{i}\right) .
$$

Finally, Lemma 2.3 ensures that $\frac{\Delta t}{\left|K_{i}\right|} \sum_{j \in \gamma(i)} \sum_{k \in \gamma(i, j)}\left|e_{j k}^{i}\right| \Phi\left(w_{i j}, w_{i k}, \nu_{j k}^{i}\right) \geq 0$ and the result is proven.

To conclude this section, we show that the reconstruction conservation property (2.19) rewrites in a very easy equivalent form as soon as a linear reconstruction is adopted. Indeed, let us consider an linear function $\widetilde{w}_{i}: K_{i} \rightarrow \mathbb{R}^{d}$ defined by

$$
\widetilde{w}_{i}(X)=w_{G_{i}}+\mu \cdot\left(X-G_{i}\right) \quad \text { with } \quad X \in K_{i},
$$

where $\mu \in \mathbb{R}^{d} \times \mathbb{R}^{d}$ is a constant given matrix. We introduce $Q_{i j}$ the middle of the edge $e_{i j}$ to set

$$
w_{i j}=\widetilde{w}_{i}\left(Q_{i j}\right)
$$

We establish that the condition (2.19) is satisfied as soon as we have

$$
w_{G_{i}}=w_{i}^{n},
$$

and reversely.

In one hand, by definition of the mass center $G_{i}$, we have $\int_{K_{i}} \widetilde{w}_{i}(X) \mathrm{d} X=\left|K_{i}\right| w_{G_{i}}$. On the other hand, the mean value of a linear function on the triangles $T_{i j}$ is given by

$$
\begin{aligned}
\int_{T_{i j}} \widetilde{w}_{i}(X) \mathrm{d} X & =\left|T_{i j}\right| \widetilde{w}_{i}\left(\frac{2}{3} Q_{i j}+\frac{1}{3} G_{i}\right), \\
& =\left|T_{i j}\right|\left(w_{G_{i}}+\frac{2}{3} \mu \cdot\left(Q_{i j}-G_{i}\right)\right), \\
& =\frac{2}{3}\left|T_{i j}\right| w_{i j}+\frac{1}{3}\left|T_{i j}\right| w_{G_{i}} .
\end{aligned}
$$

Summing over $j$, we obtain

$$
\sum_{j \in \gamma(i)}\left|T_{i j}\right| w_{i j}=\left|K_{i}\right| w_{G_{i}}
$$

and the condition (2.26) holds.

\section{THE DMGR SCHEME}

In this section, we describe the DMGR technique. The main idea is to consider two overlapping meshes, namely a primal mesh and its associated dual mesh, and to write two distinct finite volume schemes on these meshes. This process increases the number of numerical unknowns, but it allows to reconstruct very accurate gradients. First, we introduce some notations and we define the dual mesh. Next, we describe the reconstruction procedure which is performed in agreement with the assumptions made in Theorem 2.6 to enforce the required robustness. Finally we show how we take advantage of the two meshes to get states at both vertices and mass centers. 


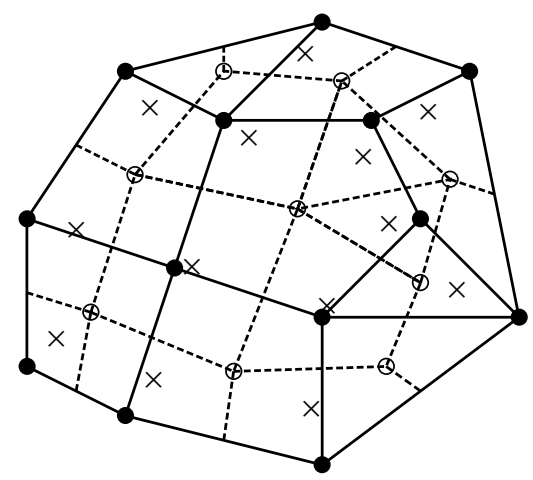

- Vertex of the primal mesh $S_{i}^{p}$

○ Center of a primal cell $G_{i}^{p}$

$=$ Vertex of the dual mesh $S_{i}^{d}$

$\times$ Center of a dual cell $G_{i}^{d}$

Figure 4. An example of a primal mesh and its associated dual mesh.

\subsection{Both primal and dual meshes}

We consider a polygonal primal mesh $\left\{K_{i}^{p}\right\}_{i \in \mathbb{Z}}$ of $\mathbb{R}^{2}$. We keep all the notations introduced in Section 2 , adding an exponent $p$ to specify we are dealing with the primal mesh. We now define a dual mesh whose cells are centered around the vertices of the primal mesh. Therefore, we denote by $\left\{S_{i}^{p}\right\}_{i \in \mathbb{Z}}$ the set of all the vertices of the primal mesh and for all $i \in \mathbb{Z}$ we set $\delta(i)$ the index set of all the cells $K_{i}^{p}$ which have $S_{i}^{p}$ as a vertex.

For each primal vertex $S_{i}^{p}$, we construct an associated dual cell $K_{i}^{d}$ obtained by joining the mass centers $\left(G_{j}^{p}\right)_{j \in \delta(i)}$ of the neighbouring cells. By construction, the vertices of the cell $K_{i}^{d}$ are thus the points $G_{j}^{p}$ with $j \in \delta(i)$. As described in [21], the cells $K_{i}^{d}$ may overlap. As a consequence, from here on we only consider primal meshes such that the cells $K_{i}^{d}$ constitute a second partition of $\mathbb{R}^{2}$, namely the dual mesh. Once again, concerning the dual mesh, we adopt the notations introduced in Section 2 by adding an exponent $d$. Let us underline that the vertices $S_{i}^{d}$ of the dual mesh coincide with the mass centers $G_{i}^{p}$ of the primal mesh. Reversely, the mass center $G_{i}^{d}$ of a dual cell is, in general, distinct from the associated primal vertex $S_{i}^{p}$ (see Fig. 4).

Provided these two meshes, we write a MUSCL scheme on each of them:

$$
\begin{aligned}
& w_{i}^{p, n+1}=w_{i}^{p, n}-\frac{\Delta t}{\left|K_{i}^{p}\right|} \sum_{j \in \gamma^{p}(i)}\left|e_{i j}^{p}\right| \phi\left(w_{i j}^{p}, w_{j i}^{p}, \nu_{i j}^{p}\right), \\
& w_{i}^{d, n+1}=w_{i}^{d, n}-\frac{\Delta t}{\left|K_{i}^{d}\right|} \sum_{j \in \gamma^{d}(i)}\left|e_{i j}^{d}\right| \phi\left(w_{i j}^{d}, w_{j i}^{d}, \nu_{i j}^{d}\right) .
\end{aligned}
$$

At this level, the derived scheme is completed as soon as the reconstructed states $w_{i j}^{p}$ and $w_{i j}^{d}$ are prescribed.

\subsection{Reconstruction process}

We now propose a reconstruction procedure over a cell based on the value of the state vector evaluated at vertices and mass center of the considered cell. Since the procedure is the same on both primal and dual cells, we detail the reconstruction in a unified way and we omit the exponent $p$ or $d$ respectively. Moreover, in this section, we assume known the value of the involved state vector at the vertices and the mass center of the adopted cell. The detail of the evaluation of $w$ at vertices and mass center will be given in the next section.

For the sake of simplicity in the notations, we here consider a cell $K$ with vertices $S_{1}, \ldots, S_{k}$. In order to adopt some simplifications, we will abusively write $S_{k+1}=S_{1}$. The mass center of the cell $K$ is denoted $G$ and, for $1 \leq i \leq k$, we introduce $T_{i+1 / 2}$ the triangle formed by the points $S_{i}, S_{i+1}$ and $G$. At last, $Q_{i+1 / 2}$ is the middle of the edge $S_{i} S_{i+1}$ (see Fig. 5).

We assume known the state vectors $w_{i} \in \Omega$ at each vertex $S_{i}$, as well as the state vector $w_{0} \in \Omega$ at the mass center $G$. The aim is to reconstruct a state vector $\widehat{w}_{i+1 / 2}$ at each point $Q_{i+1 / 2}$. The reader is referred to Figure 5 for an illustration of the locations of the known states and the reconstructed states. 

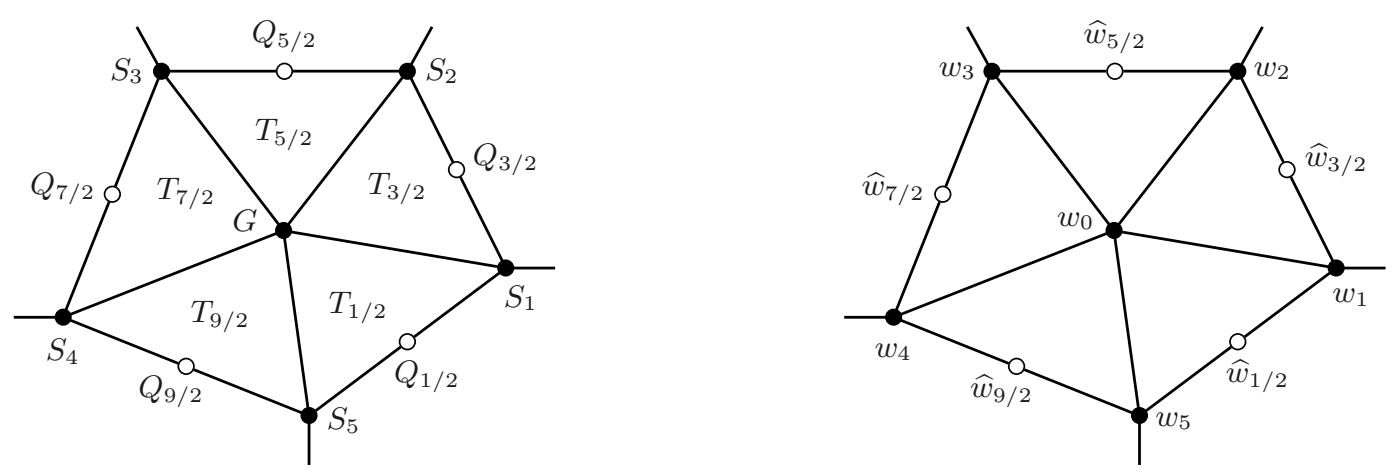

FiguRE 5. Left: geometry of the cell K. Right: known states (black dots) and unknown reconstructed states (white dots).

Here, we suggest linear reconstructions. According to (2.25) and (2.26), the assumption (2.19) of Theorem 2.6 will be satisfied as soon as the considered linear reconstruction $\widetilde{w}_{\mu}: K \rightarrow \mathbb{R}^{d}$ writes as follows:

$$
\widetilde{w}_{\mu}(X)=w_{0}+\mu \cdot(X-G),
$$

where $\mu$ stands for an accurate approximation of the space gradient of the solution. To evaluate $\mu$, a three-step procedure is adopted.

First step: Gradient reconstruction.

There exists a unique linear function $\bar{w}_{i+1 / 2}: T_{i+1 / 2} \mapsto \mathbb{R}^{d}$ such that $\bar{w}_{i+1 / 2}\left(S_{i}\right)=w_{i}, \bar{w}_{i+1 / 2}\left(S_{i+1}\right)=w_{i+1}$ and $\bar{w}_{i+1 / 2}(G)=w_{0}$. Next, let us introduce $\bar{w}: K \mapsto \mathbb{R}^{d}$, the continuous piecewise linear function defined by

$$
\bar{w}(X)=\bar{w}_{i+1 / 2}(X), \quad \forall X \in T_{i+1 / 2} .
$$

Second step: Projection.

We define $\widetilde{w}_{\mu}: K \rightarrow \mathbb{R}^{d}$, the linear function resulting from the $L^{2}$-projection of $\bar{w}$ as follows:

$$
\int_{K}\left\|\bar{w}(X)-\widetilde{w}_{\mu}(X)\right\|^{2} \mathrm{~d} X=\min _{\alpha \in \mathbb{R}^{d} \times \mathbb{R}^{d}} \int_{K}\left\|\bar{w}(X)-\widetilde{w}_{\alpha}(X)\right\|^{2} \mathrm{~d} X .
$$

By involving standard convex arguments, we easily have the existence of a unique $\mu$. Let us underline that the numerical evaluation of $\mu$ straightforwardly comes from the minimum of a quadratic function.

Third step: Limitation of the slopes.

At this level, we cannot directly consider the reconstructed states given by $\widehat{w}_{i+1 / 2}=\widetilde{w}_{\mu}\left(Q_{i+1 / 2}\right)$. Indeed, the reconstruction coming from the above projection-step does not necessarily belong to $\Omega$. To enforce the reconstruction to be $\Omega$-preserving, we suggest multiplying the slopes by a limiter. Let us consider the sets of admissible slope limiters as follows:

$$
F_{i+1 / 2}=\left\{\theta \in[0,1] / \widetilde{w}_{s \mu}\left(Q_{i+1 / 2}\right) \in \Omega, \forall s \in[0, \theta]\right\} .
$$

Since for all $0 \leq i \leq k-1$ we have $\widetilde{w}_{0}\left(Q_{i+1 / 2}\right)=w_{0} \in \Omega$, none of the sets $F_{i+1 / 2}$ is empty. We define the optimal slope limiter as follows:

$$
\beta=\min _{0 \leq i \leq k-1} \sup \left(F_{i+1 / 2}\right)-\epsilon
$$

where $\epsilon>0$ is a fixed parameter such that $\beta \in \bigcap_{i} F_{i+1 / 2}$. As a consequence, we obtain $\widetilde{w}_{\beta \mu}\left(Q_{i+1 / 2}\right) \in \Omega$, for all $0 \leq i \leq k-1$.

The required reconstructed states are given by $\widehat{w}_{i+1 / 2}=\widetilde{w}_{\beta \mu}\left(Q_{i+1 / 2}\right)$. By construction, they satisfy the hypotheses of Theorem 2.6 and therefore the DMGR scheme is $\Omega$-preserving under the CFL condition $(2.20)$. 


\subsection{State evaluations at the vertices and at the mass centers}

To conclude the presentation of the reconstruction procedure, we have to specify the evaluation of the states at the vertices and at the mass centers of both primal and dual cells. The primal and dual mass center states are given by the numerical schemes (3.1)-(3.2). In addition, the vertices of the dual mesh exactly coincide with the mass centers of the primal mesh. As a consequence, the values at a dual vertex are given by the values at the mass center of the associated primal cell (see Fig. 5).

At this level, the reconstruction procedure on the dual cells can be applied. Hence, on each cell $K_{i}^{d}$, we can characterized the linear function $\widetilde{w}_{i}^{d}$.

Next, to define the primal reconstruction $\widetilde{w}_{i}^{p}$, we need an evaluation of the state vector at vertex $S_{i}^{p}$. As mentioned before, a vertex $S_{i}^{p}$ of the primal mesh do not necessarily coincide with the mass center $G_{i}^{d}$ of the associated dual cell. A basic first choice for the state at the point $S_{i}^{p}$ would be to consider the value at the mass center of the associated dual cell $w_{i}^{d, n}$. However this turns out to be a too coarse approximation, mainly with very distorted meshes. Since the dual reconstruction $\widetilde{w}_{i}^{d}$ is known, we suggest to consider $\widetilde{w}_{i}^{d}\left(S_{i}^{p}\right)$ as the state value at the point $S_{i}^{p}$ which will be a fine approximation as illustrated in the numerical experiments.

\section{Numerical Results}

The DMGR scheme has been implemented into an unstructured code to approximate the weak solutions of the 2D Euler equations:

$$
\partial_{t}\left(\begin{array}{c}
\rho \\
\rho u \\
\rho v \\
E
\end{array}\right)+\partial_{x}\left(\begin{array}{c}
\rho u \\
\rho u^{2}+p \\
\rho u v \\
u(E+p)
\end{array}\right)+\partial_{y}\left(\begin{array}{c}
\rho v \\
\rho u v \\
\rho v^{2}+p \\
v(E+p)
\end{array}\right)=0,
$$

where $\rho,(u, v), E$ and $p$ are the density, the velocity, the total energy and the pressure respectively. The system is closed by the perfect gas law

$$
p=(\gamma-1)\left(E-\rho \frac{u^{2}+v^{2}}{2}\right)
$$

with $\gamma \in(1,3]$ the adiabatic coefficient. In the following numerical experiments, $\gamma$ will be fixed to 1.4. The set of admissible states is defined by

$$
\Omega=\left\{w=(\rho, \rho u, \rho v, E)^{T} \in \mathbb{R}^{4}, \rho>0, E-\frac{\rho}{2}\left(u^{2}+v^{2}\right)>0\right\} .
$$

We have performed several classical test cases to assess the accuracy and the stability of the method. First, we consider an isentropic vortex to evaluate the errors and the numerical order of convergence. Indeed, the exact solution of the isentropic vortex is known and smooth enough. Then we proceed with a 2D Riemann problem as proposed in [30]. We conclude the series of test with two classical experiments, the double Mach reflection on a ramp and the Mach 3 wind tunnel with a step. These problems emphasize the ability of the DMGR scheme to accurately capture strong shocks and contact discontinuities. In addition, the Double Mach reflection on a ramp benchmark is considered to performed several comparisons between the DMGR scheme and a classical MUSCL reconstruction.

For all tests, the adopted numerical flux function is given by the Suliciu relaxation scheme $[8,15]$. We systematically compare the results obtained by the first-order scheme and by the DMGR scheme. Concerning the time step restriction, we adopt the derived CFL conditions (2.13) or (2.20) according to the order of accuracy. In order to illustrate the efficiency of the DMGR spatial discretization, we only consider a first-order time approximation. In order to make a fair comparison, we have to perform the simulations with a number of degrees of freedom (DOF) of the same order. The number of DOF is the number of numerical unknowns of the scheme: for the first-order scheme and for the classical MUSCL scheme, it is simply the number of cells of the mesh, while for the DMGR scheme, it is the number of cells of the primal mesh plus the number of cells of the dual mesh. 


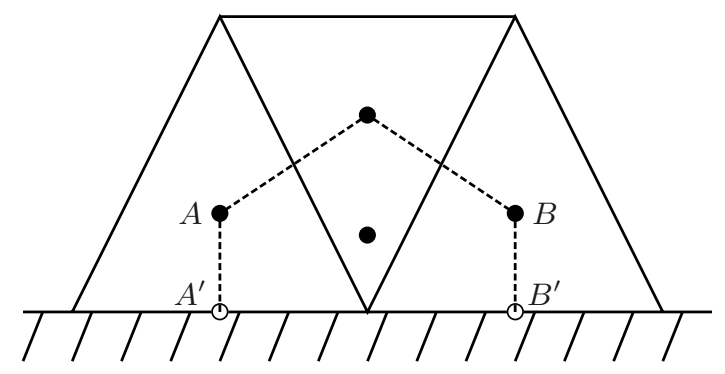

Figure 6. Reconstruction on a boundary dual cell. Black dots: known states, white dots: unknown states.

To deal with the boundary conditions, the main difficulty comes from the reconstruction on boundary cells. Indeed, as soon as the reconstruction at the boundary is known, the boundary flux is evaluated as in usual finite volume methods. First, let us deal with a dual boundary cell as illustrated on Figure 6. In order to apply our DMGR reconstruction technique, the unknown state vectors at points $A^{\prime}$ and $B^{\prime}$ are missing and must be evaluated. To address such an issue, several interpolation techniques can be considered. We adopt the simplest one given by $w\left(A^{\prime}\right)=w(A)$ and $w\left(B^{\prime}\right)=w(B)$. At this level, the reconstruction is achieved on the dual cells and it remains to deal with the primal cells. We easily note that the primal cell reconstruction procedure introduced in Section 3.3 can be directly applied.

\subsection{Isentropic vortex}

The isentropic vortex problem is presented in $[20,42]$. Let us consider a mean flow characterized by $\rho_{\infty}=1$, $\left(u_{\infty}, v_{\infty}\right)=(1,1)$ and $p_{\infty}=1$. We add to this mean flow an isentropic vortex, centered at the point $(0,0)$, which is defined by perturbations in velocity and temperature $T=\frac{p}{\rho}$, but which do not involve perturbation for entropy $S=\frac{p}{\rho^{\gamma}}$. The vortex is given by the conditions:

$$
\begin{gathered}
(\delta u, \delta v)=\frac{\epsilon}{2 \pi} \exp \left(\frac{1-r^{2}}{2}\right)(-y, x), \\
\delta T=-\frac{(\gamma-1) \epsilon^{2}}{8 \gamma \pi^{2}} \exp \left(1-r^{2}\right), \quad \delta S=0,
\end{gathered}
$$

where $r^{2}=x^{2}+y^{2}$ and the vortex strength is set at $\epsilon=5$. The initial density and pressure are thus given by:

$$
\begin{gathered}
\rho=\rho_{\infty}\left(\frac{T}{T_{\infty}}\right)^{\frac{1}{\gamma-1}}=\left(1-\frac{(\gamma-1) \epsilon^{2}}{8 \gamma \pi^{2}} \exp \left(1-r^{2}\right)\right)^{\frac{1}{\gamma-1}}, \\
p=\rho T=\left(1-\frac{(\gamma-1) \epsilon^{2}}{8 \gamma \pi^{2}} \exp \left(1-r^{2}\right)\right)^{\frac{\gamma}{\gamma-1}} .
\end{gathered}
$$

The computational domain is given by $[-5,5] \times[-5,5]$ and we impose periodic boundary conditions. We can easily check that the exact solution is the convection of the initial vortex at the mean velocity.

In order to estimate the numerical convergence order of the DMGR scheme, we compute the numerical solution on a series of refined Cartesian meshes. The simulations are stopped at time $t=10$, after one period, which means that the exact solutions at final time and at initial time coincide. We evaluate the numerical relative $L^{1}$ and $L^{\infty}$ errors for the density as follows:

$$
\operatorname{err}_{1}=\frac{\sum_{i}\left|\rho_{i}^{N}-\rho_{i}^{0}\right|\left|K_{i}\right|}{\sum_{i}\left|\rho_{i}^{0}\right|\left|K_{i}\right|} \text { and } \operatorname{err} r_{\infty}=\frac{\max _{i}\left|\rho_{i}^{N}-\rho_{i}^{0}\right|}{\max _{i}\left|\rho_{i}^{0}\right|}
$$

where $\left(\rho_{i}^{0}\right)_{i}$ and $\left(\rho_{i}^{N}\right)_{i}$ are respectively the primal cell mean density values at initial time and at final time. 
TABLE 1. $L^{1}$ and $L^{\infty}$ errors and convergence rates for the isentropic vortex problem with the first-order scheme and the DMGR scheme.

\begin{tabular}{lccccccccc}
\hline \multicolumn{4}{c}{ First-order scheme } & \multicolumn{5}{c}{ DMGR scheme } \\
\hline Nb DOF & $L^{1}$ error & \multicolumn{1}{c}{$L^{\infty}$ error } & & Nb DOF & $L^{1}$ error & \multicolumn{3}{c}{$L^{\infty}$ error } \\
\hline 841 & $2.5718 \mathrm{E}-02$ & - & $4.5570 \mathrm{E}-01$ & - & 841 & $1.3296 \mathrm{E}-02$ & - & $2.6987 \mathrm{E}-01$ & - \\
1600 & $2.4529 \mathrm{E}-02$ & 0.15 & $4.3359 \mathrm{E}-01$ & 0.15 & 3281 & $4.5387 \mathrm{E}-03$ & 1.58 & $9.5782 \mathrm{E}-02$ & 1.52 \\
6400 & $2.0611 \mathrm{E}-02$ & 0.25 & $3.9529 \mathrm{E}-01$ & 0.13 & 12961 & $1.3228 \mathrm{E}-03$ & 1.79 & $2.6453 \mathrm{E}-02$ & 1.87 \\
25600 & $1.5421 \mathrm{E}-02$ & 0.42 & $3.0631 \mathrm{E}-01$ & 0.37 & 52480 & $3.5605 \mathrm{E}-04$ & 1.88 & $6.4104 \mathrm{E}-03$ & 2.03 \\
102400 & $1.0073 \mathrm{E}-02$ & 0.61 & $1.9370 \mathrm{E}-01$ & 0.66 & 205541 & $9.3363 \mathrm{E}-05$ & 1.96 & $1.5533 \mathrm{E}-03$ & 2.08 \\
409600 & $5.9059 \mathrm{E}-03$ & 0.77 & $1.0518 \mathrm{E}-01$ & 0.88 & 409513 & $4.7932 \mathrm{E}-05$ & 1.93 & $7.8221 \mathrm{E}-04$ & 1.99 \\
\hline
\end{tabular}
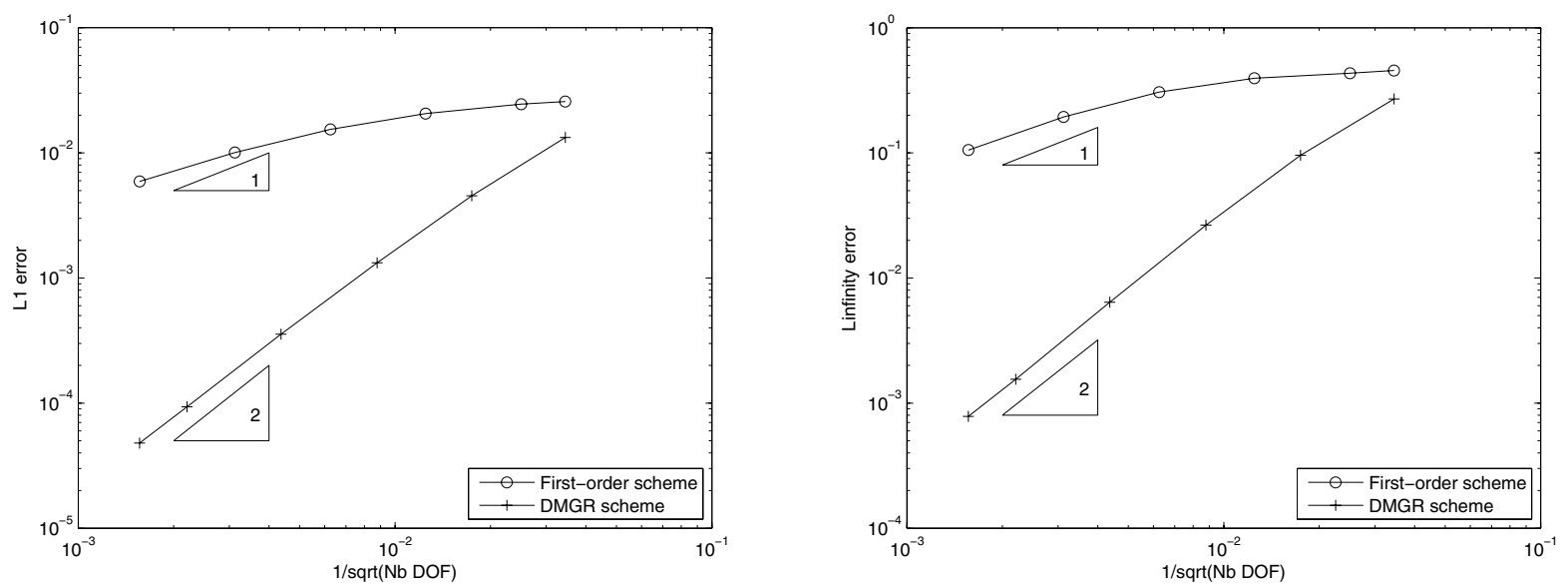

FiguRE 7. Convergence curves for the isentropic vortex approximated by the first-order scheme and the DMGR scheme. Left: $L^{1}$ error. Right: $L^{\infty}$ error.

In Table 1, the $L^{1}$ and $L^{\infty}$ errors and rates of convergence for the first-order scheme and the DMGR scheme are given. We observe that second-order is effectively reached for the DMGR scheme. The corresponding convergence curves are displayed in Figure 7.

\subsection{D Riemann problem}

We now turn simulating one of the 2D Riemann problems as proposed in [30]. The computational domain is $[0,1] \times[0,1]$ divided into four quadrants by the lines $x=1 / 2$ and $y=1 / 2$. The $2 \mathrm{D}$ Riemann problems are defined by initial constant states on each quadrant given in Table 2.

In order to simplify the boundary condition evaluations, we choose our primal mesh as described Figure 8.

We perform the test using about $1.5 \times 10^{6}$ DOF with both the first-order scheme and the DMGR scheme. The final computational time is $t=0.3$. We show a zoom on the complex structure developing in the center in Figure 9 . We clearly note the improvement involved by the DMGR approach and the ability of the scheme to develop Kelvin-Helmotz instabilities. 
TABLE 2. Initial states of the 2D Riemann problem in the four quadrants for the density $\rho$, the velocity $(u, v)$ and the pressure $p$.

\begin{tabular}{ccccccccc}
\hline & \multicolumn{4}{c}{ left } & \multicolumn{5}{c}{ right } \\
& $\rho$ & $u$ & $v$ & $p$ & $\rho$ & $u$ & $v$ & $p$ \\
\hline Upper & 0.5323 & 1.206 & 0.0 & 0.3 & 1.5 & 0.0 & 0.0 & 1.5 \\
Lower & 0.138 & 1.206 & 1.206 & 0.029 & 0.5323 & 0.0 & 1.206 & 0.3 \\
\hline
\end{tabular}

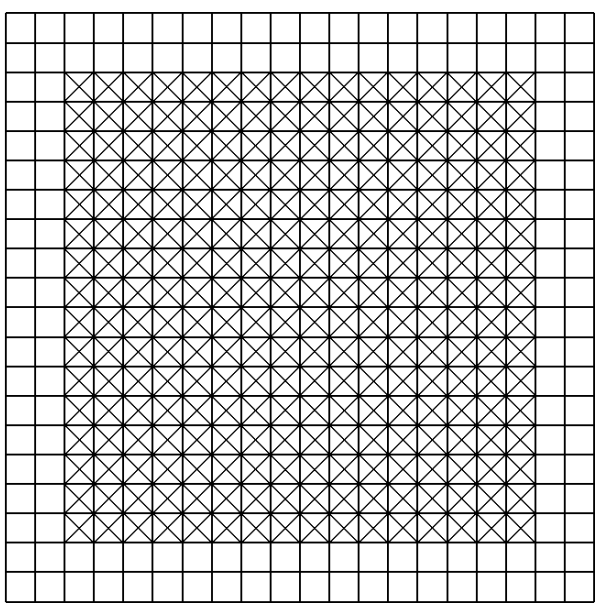

Figure 8. Primal mesh used for the 2D Riemann problem.
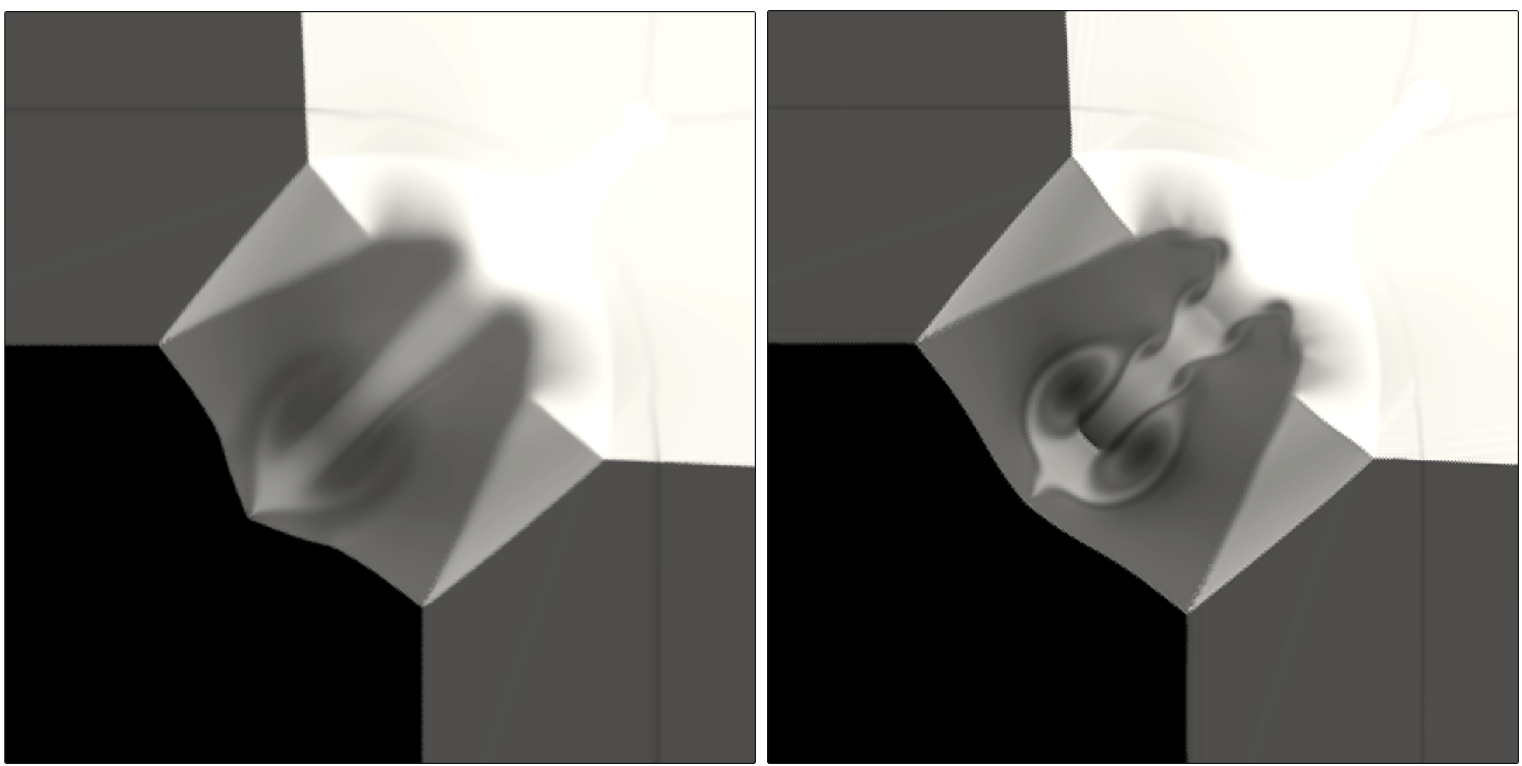

Figure 9. Density solution of the 2D Riemann problem using $1.5 \times 10^{6}$ DOF - Zoom on the square $[0.15,0.55]^{2}-$ Left: first-order approximation - Right: DMGR approximation. 


\subsection{Double Mach reflection on a ramp}

The next experiment concerns the double Mach reflection on a ramp as proposed by Woodward and Colella in [46] (see also [6,41]). It involves strong shocks and a very complex structure due to contact discontinuities. This test consists of the interaction of a planar Mach 10 shock with a ramp making a $30^{\circ}$ angle with the $x$-axis.

We assume that the whole computation domain have the following initial condition: $\rho=1.4,(u, v)=(0,0)$, $p=1$. We assign reflexive boundary condition to the bottom and the top boundaries. We impose an inflow condition given by $\rho=8,(u, v)=(8.25,0)$ and $p=116.5$ on the left side in order to create the Mach 10 shock and an outflow boundary condition on the right side to let the gas break out. We stop the simulation at time $T=0.2$, before the structure reaches the top boundary.

The test is performed using an unstructured mesh about $3 \times 10^{6}$ DOF. Figure 10 depicts 30 density isolines obtained by the first-order scheme and both second-order DMGR scheme and usual MUSCL scheme. In addition, a zoom (made of 50 density isolines) on the wave interaction area is also displayed. In order to make relevant the second-order scheme comparison, we use the limiter introduced by Barth and Jespersen [3]. This limiter is widely used in the literature (for instance, see $[19,29,37]$ ). The main shock wave is connected to a triple junction point with an incident and reflected shock wave. Issuing from the triple point, a slip line appears behind the incident wave. Another shock wave appears inside the wave structure coming from the main shock to interact with the slip line.

As expected, the DMGR scheme captures very accurately this sophisticated wave structure. In addition, the slip line corresponds to a contact discontinuity and we can see that Kelvin-Helmholtz instabilities are generated. Let us underline that classical MUSCL schemes are not able to capture such small structures. As mentioned by [41], the appearance of such small structures in the flow points out the smallness of the inherent numerical viscosities of the DMGR scheme.

Concerning the efficiency of the DMGR method, this numerical experiment needs 62672 time iterations which represents a physical mean time step given by $\Delta t=3.2 \times 10^{-6}$. The computation was performed using a parallel code for a CPU time of $8 \mathrm{~h} 49$. As a comparison, the standard MUSCL scheme with the same number of DOF uses $9 \mathrm{~h} 02$ of CPU time. This numerical experiment was achieved with 78453 time iterations, which corresponds to a physical mean time step $\Delta t=2.5 \times 10^{-6}$. We note that the DMGR scheme is less CFL restrictive than the classical MUSCL approach since when we consider the same number of DOF, our method involves larger cells. Indeed, to get $3 \times 10^{6} \mathrm{DOF}$, the DMGR method is based on a primal mesh made of $2 \times 10^{6}$ cells and a dual mesh made of $1 \times 10^{6}$ cells, whereas the MUSCL technique needs a mesh made of $3 \times 10^{6}$ cells. This explains that the cells involved by the MUSCL scheme are smaller than the cells involved by the DMGR scheme. However it seems that each cell computation is a little more expensive for the DMGR reconstruction than for the MUSCL reconstruction. In conclusion, both methods have a similar CPU time cost, but with better accuracy for the DMGR technique.

\subsection{Mach 3 wind tunnel with a step}

The last numerical test is devoted to the Mach 3 wind tunnel with a step, initially proposed in [46]. The wind tunnel is 1 length unit wide and 3 length units long. The step is located 0.6 length units from the left-hand side of the domain and is 0.2 length units high. Initially, the wind tunnel is filled with a gas with constant density $\rho=1.4$, pressure $p=1$ and velocity $(u, v)=(3,0)$, describing a uniform Mach 3 flow. Along the walls of the tunnel (and in front of the step), reflective boundary conditions are prescribed. The same Mach 3 flow is applied as an inflow condition on the left side and an outflow boundary condition is set on the right side.

Unstructured meshes made of about $1.5 \times 10^{6} \mathrm{DOF}$ are used to compute this test case. Numerical results are displayed in Figure 11 at time $t=4$ using 30 density isolines with both first-order scheme and DMGR scheme.

Once again, the DMGR scheme gives very good results. First of all, we notice Kelvin-Helmotlz instabilities appearing from the upper slip line. Next, concerning the triple point, the first-order approximation locate it near the step interface while, as mentioned by [14], it should be exactly located on the step interface. According to Figure 12, this location default of the triple point is corrected by the DMGR scheme. However we note 

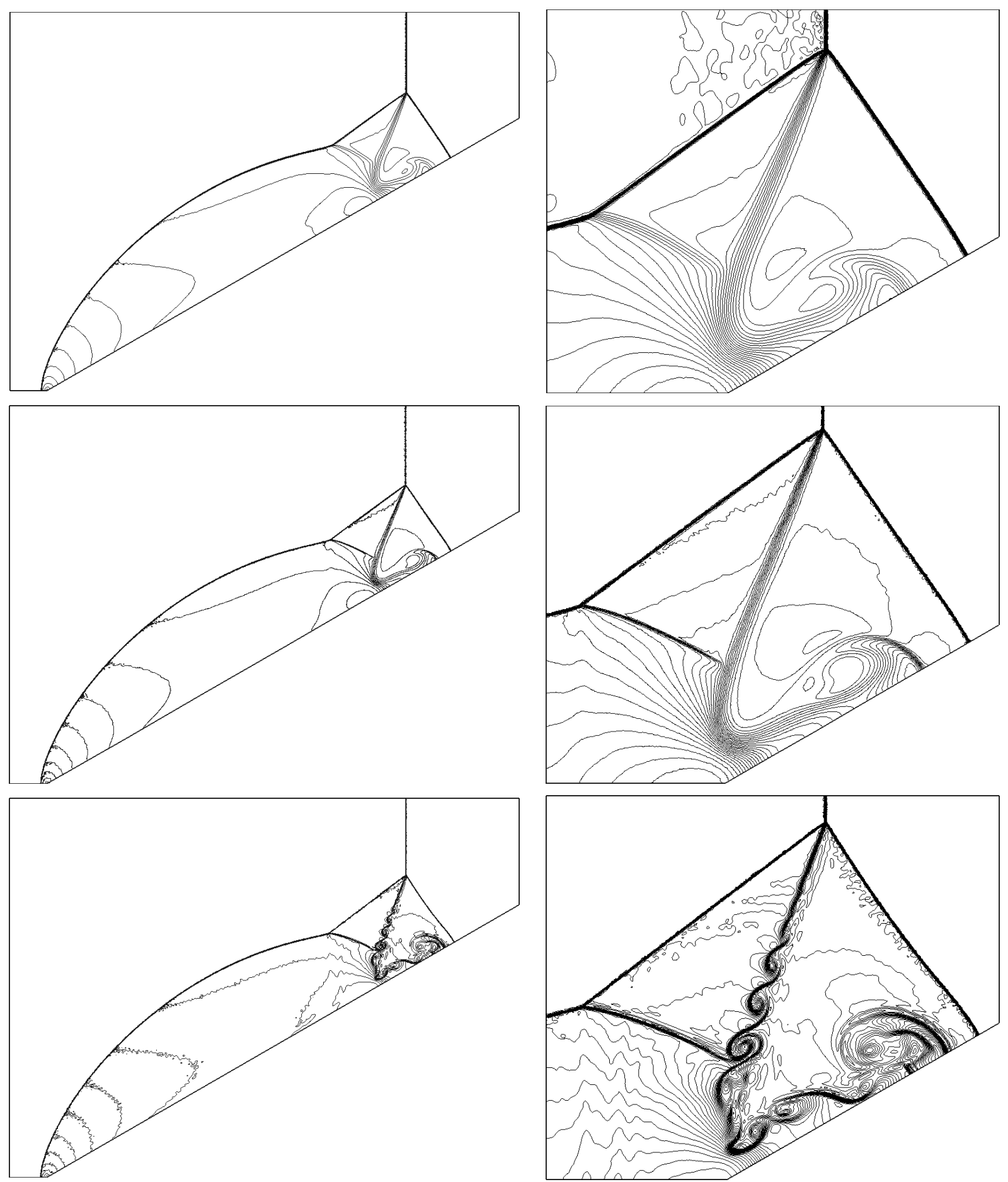

Figure 10. Double Mach reflection on a ramp with $3 \times 10^{6}$ DOF - On the left, full computational domain with 30 density isolines. On the right, zoom on the wave interaction area with 50 density isolines - Top: first-order approximation. Middle: classical MUSCL approximation. Bottom: DMGR approximation. 


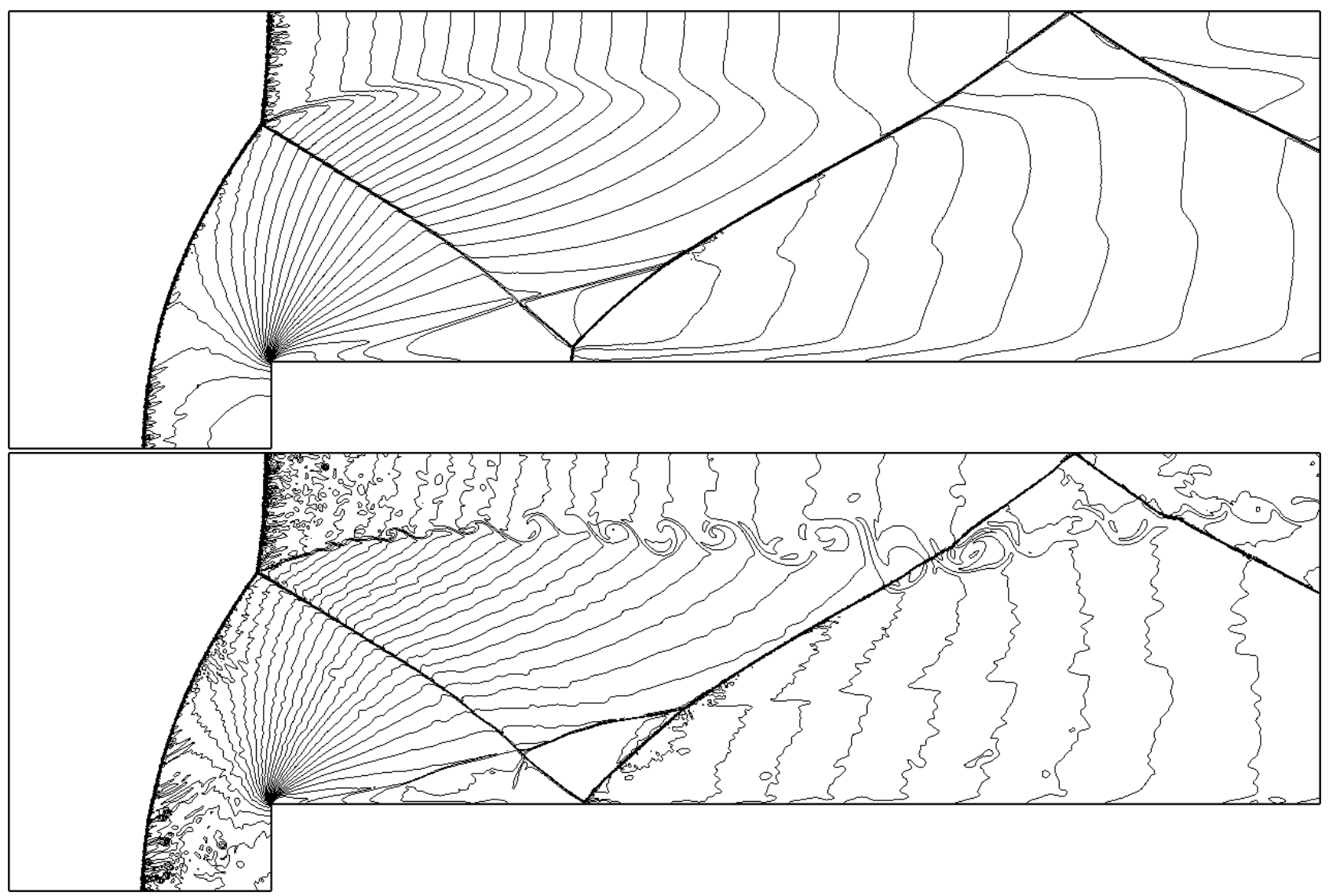

FiguRE 11. Mach 3 wind tunnel with a step with $1.5 \times 10^{6}$ DOF -30 density isolines - Top: first-order approximation - Bottom: DMGR approximation.

some oscillations arising behind the first shock. This is probably due to the lack of a TVD-like criterion in the presented DMGR reconstruction technique.

\section{Conclusion}

In the present work, we have presented a new strategy to approximate the gradient of the computed solution. This procedure stays free from the definition of the mesh (structured or unstructured) and it allows us to introduce an improvement of the so-called MUSCL schemes. To address such an issue, we have built a dual mesh. By considering simultaneously both primal and dual meshes, we are able to accurately predict the gradient of the solution. This process increases the number of DOF compared with the classical MUSCL schemes, but it remains lower than the number of DOF of the $P^{1}$-discontinuous-Galerkin method. Indeed, on a triangular mesh with $N$ cells, the number of DOF for the $P^{1}$-discontinuous-Galerkin method is $3 N$, while it is about $3 N / 2$ for the DMGR scheme.

Concerning 3D extensions, the main difficulty comes from the derivation of a suitable dual mesh. Several options were developed in the framework of the DDFV method to approximate the solutions of elliptic/parabolic equations. For instance, in $[1,17]$, the strategy lies on a localization of the unknowns at the cell centers and at the vertices. Supplementary unknowns can be considered either at the face centers [26] or at the middle of the 

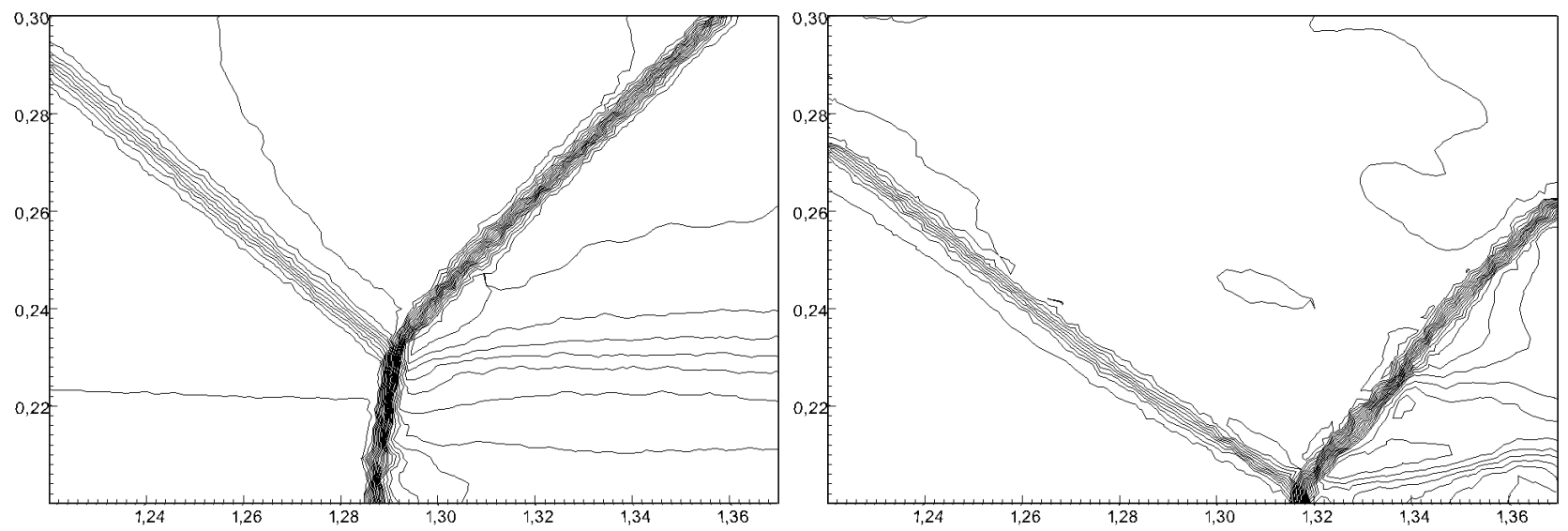

Figure 12. Mach 3 wind tunnel with a step with $1.5 \times 10^{6}$ DOF - Zoom on the triple point location - 30 density isolines - Left: first-order approximation - Right: DMGR approximation.

edges [16]. It is possible to adapt these 3D DDFV techniques to generalize our DMGR reconstruction technique to $3 \mathrm{D}$ problems.

In addition we have proved the robustness of the suggested strategy by introducing suitable CFL like condition. Accordingly to several recent works, the obtained second-order CFL is surely not optimal since it does not restore the first-order CFL as soon as the MUSCL reconstruction vanishes. However the performed numerical simulations have shown a very good behaviour of the approximations.

Acknowledgements. The authors would like to thank Guy Moebs of the CCIPL (http://www.ccipl.univ-nantes.fr/) for his help in optimizing the code.

\section{REFERENCES}

[1] B. Andreianov, M. Bendahmane and K.H. Karlsen, Discrete duality finite volume schemes for doubly nonlinear degenerate hyperbolic-parabolic equations. J. Hyperbolic Differ. Equ. 7 (2010) 1-67.

[2] B. Andreianov, F. Boyer and F. Hubert, Discrete duality finite volume schemes for Leray-Lions-type elliptic problems on general 2D meshes. Numer. Methods Partial Differ. Equ. 23 (2007) 145-195.

[3] T. Barth and D. Jespersen, The design and application of upwind schemes on unstructured meshes, in AIAA, Aerospace Sciences Meeting, 27 th, Reno, NV(1989).

[4] M. Berger, M.J. Aftosmis and S.M. Murman, Analysis of slope limiters on irregular grids, in 43rd AIAA Aerospace Sciences Meeting, volume NAS Technical Report NAS-05-007 (2005).

[5] C. Berthon, Stability of the MUSCL schemes for the Euler equations. Commun. Math. Sci. 3 (2005) 133-158.

[6] C. Berthon, Numerical approximations of the 10-moment Gaussian closure. Math. Comput. 75 (2006) $1809-1832$.

[7] C. Berthon, Robustness of MUSCL schemes for 2D unstructured meshes. J. Comput. Phys. 218 (2006) $495-509$.

[8] F. Bouchut, Nonlinear stability of finite volume methods for hyperbolic conservation laws and well-balanced schemes for sources. Frontiers in Mathematics. Birkhäuser Verlag, Basel (2004).

[9] F. Bouchut, C. Bourdarias and B. Perthame, A MUSCL method satisfying all the numerical entropy inequalities. Math. Comput. 65 (1996) 1439-1462.

[10] T. Buffard and S. Clain, Monoslope and multislope MUSCL methods for unstructured meshes. J. Comput. Phys. 229 (2010) 3745-3776.

[11] C. Calgaro, E. Chane-Kane, E. Creusé and T. Goudon, L $^{\infty}$-stability of vertex-based MUSCL finite volume schemes on unstructured grids: Simulation of incompressible flows with high density ratios. J. Comput. Phys. 229 (2010) 6027-6046.

[12] C. Calgaro, E. Creusé, T. Goudon and Y. Penel, Positivity-preserving schemes for Euler equations: sharp and practical CFL conditions (2012). preprint.

[13] S. Clain and V. Clauzon, L ${ }^{\infty}$ stability of the MUSCL methods. Numerische Mathematik 116 (2010) 31-64.

[14] S. Clain, S. Diot and R. Loubère, A high-order finite volume method for hyperbolic systems: Multi-dimensional Optimal Order Detection (MOOD). J. Comput. Phys. (2011). 
[15] F. Coquel and B. Perthame, Relaxation of Energy and Approximate Riemann Solvers for General Pressure Laws in Fluid Dynamics. SIAM J. Numer. Anal. 35 (1998) 2223-2249.

[16] Y. Coudière and F. Hubert, A 3d discrete duality finite volume method for nonlinear elliptic equations. SIAM J. Sci. Comput. 33 (2011) 1739-1764.

[17] Y. Coudière, C. Pierre, O. Rousseau and R. Turpault, A 2D/3D discrete duality finite volume scheme. Application to ECG simulation. Int. J. Finite 6 (2009) 24.

[18] P.H. Cournède, B. Koobus and A. Dervieux, Positivity statements for a mixed-element-volume scheme on fixed and moving grids. European J. Comput. Mechanics/Revue Européenne de Mécanique Numérique 15 (2006) 767-798.

[19] M.S. Darwish and F. Moukalled, Tvd schemes for unstructured grids. International Journal of Heat and Mass Transfer 46 (2003) 599-611.

[20] S. Diot, S. Clain, R. Loubère, Improved Detection Criteria for the Multi-dimensional Optimal Order Detection (MOOD) on unstructured meshes with very high-order polynomials. Comput. Fluids 64 (2012) 43-63.

[21] K. Domelevo and P. Omnes, A finite volume method for the Laplace equation on almost arbitrary two-dimensional grids. Math. Model. Numer. Anal. 39 (2005) 1203-1249.

[22] R. Ghostine, G. Kesserwani, R. Mosé, J. Vazquez and A. Ghenaim, An improvement of classical slope limiters for high-order discontinuous Galerkin method. Internat. J. Numer. Methods Fluids 59 (2009) 423-442.

[23] E. Godlewski and P.A. Raviart, Numerical approximation of hyperbolic systems of conservation laws, in vol. 118 of Appl. Math. Sci. Springer-Verlag, New York (1996).

[24] A. Harten, P.D. Lax and B. Van Leer, On upstream differencing and Godunov-type schemes for hyperbolic conservation laws. SIAM Review (1983) 35-61.

[25] F. Hermeline, A finite volume method for the approximation of diffusion operators on distorted meshes. J. Comput. Phys. 160 (2000) 481-499.

[26] F. Hermeline, Approximation of 2-D and 3-D diffusion operators with variable full tensor coefficients on arbitrary meshes. Comput. Methods Appl. Mech. Engrg. 196 (2007) 2497-2526.

[27] M.E. Hubbard, Multidimensional slope limiters for MUSCL-type finite volume schemes on unstructured grids. J. Comput. Phys. 155 (1999) 54-74.

[28] B. Keen and S. Karni, A second order kinetic scheme for gas dynamics on arbitrary grids. J. Comput. Phys. 205 (2005) $108-130$.

[29] K. Kitamura and E. Shima, Simple and parameter-free second slope limiter for unstructured grid aerodynamic simulations. AIAA J. 50 (2012) 1415-1426.

[30] A. Kurganov and E. Tadmor, Solution of two-dimensional Riemann problems for gas dynamics without Riemann problem solvers. Numer. Methods Partial Differ. Equ. 18 (2002) 584-608.

[31] P.D. Lax, Shock waves and entropy, in Contributions to nonlinear functional analysis (Proc. Sympos., Math. Res. Center, Univ. Wisconsin, Madison, Wis., 1971). Academic Press, New York (1971) 603-634.

[32] P.D. Lax, Hyperbolic systems of conservation laws and the mathematical theory of shock waves. Conference Board of the Math. Sci. Regional Conference Series Appl. Math. No. 11. Society for Industrial and Applied Mathematics, Philadelphia, Pa. (1973).

[33] R.J. LeVeque, Finite volume methods for hyperbolic problems. Cambridge Univ Press (2002).

[34] Wanai Li, Yu-Xin Ren, Guodong Lei and Hong Luo, The multi-dimensional limiters for solving hyperbolic conservation laws on unstructured grids. J. Comput. Phys. 230 (2011) 7775-7795.

[35] Q. Liang and F. Marche, Numerical resolution of well-balanced shallow water equations with complex source terms. Advances in Water Resources 32 (2009) 873-884.

[36] X.D. Liu, A maximum principle satisfying modification of triangle based adaptive stencils for the solution of scalar hyperbolic conservation laws. SIAM J. Numer. Anal. (1993) 701-716.

[37] K. Michalak and C. Ollivier-Gooch, Limiters for unstructured higher-order accurate solutions of the euler equations, in Proc. of the AIAA Forty-sixth Aerospace Sciences Meeting (2008).

[38] B. Perthame, Second-order Boltzmann schemes for compressible Euler equations in one and two space dimensions. SIAM J. Numer. Anal. (1992) 1-19.

[39] B. Perthame and Y. Qiu, A variant of Van Leer's method for multidimensional systems of conservation laws. J. Computat. Phys. 112 (1994) 370-381.

[40] B. Perthame and C.W. Shu, On positivity preserving finite volume schemes for Euler equations. Numerische Mathematik $\mathbf{7 3}$ (1996) 119-130.

[41] J. Shi, Y.T. Zhang and C.W. Shu, Resolution of high order WENO schemes for complicated flow structures. J. Comput. Phys. 186 (2003) 690-696.

[42] C.W. Shu, Essentially non-oscillatory and weighted essentially non-oscillatory schemes for hyperbolic conservation laws. Advanced numerical approximation nonlinear Hyperbolic equations (1998) 325-432.

[43] E.F. Toro, Riemann solvers and numerical methods for fluid dynamics: a practical introduction. Springer Verlag (2009).

[44] B. Van Leer, Towards the ultimate conservative difference scheme. V. A second-order sequel to Godunov's method. J. Comput. Phys. 32 (1979) 101-136.

[45] V Venkatakrishnan, Convergence to steady state solutions of the euler equations on unstructured grids with limiters. J. Comput. Phys. 118 (1995) 120-130.

[46] P. Woodward and P. Colella, The numerical simulation of two-dimensional fluid flow with strong shocks. J. Comput. Phys. 54 (1984) 115-173. 